\title{
Perineuronal net degradation rescues CA2 plasticity in a mouse model of Rett syndrome
}

\author{
Kelly E. Carstens, Daniel J. Lustberg, Emma K. Shaughnessy, Katharine E. McCann, Georgia M. Alexander, and Serena M. Dudek
}

Neurobiology Laboratory, National Institute of Environmental Health Sciences, NIH, Research Triangle Park, North Carolina, USA.

\begin{abstract}
Perineuronal nets (PNNs), a specialized form of extracellular matrix, are abnormal in the brains of people with Rett syndrome (RTT). We previously reported that PNNs function to restrict synaptic plasticity in hippocampal area CA2, which is unusually resistant to long-term potentiation (LTP) and has been linked to social learning in mice. Here we report that PNNs appear elevated in area CA2 of the hippocampus of an individual with RTT and that PNNs develop precociously and remain elevated in area CA2 of a mouse model of RTT (Mecp2-null). Further, we provide evidence that LTP could be induced at CA2 synapses prior to PNN maturation (postnatal day 8-11) in wild-type mice and that this window of plasticity was prematurely restricted at CA2 synapses in Mecp2-null mice. Degrading PNNs in Mecp2-null hippocampus was sufficient to rescue the premature disruption of CA2 plasticity. We identified several molecular targets that were altered in the developing Mecp2-null hippocampus that may explain aberrant PNNs and CA2 plasticity, and we discovered that CA2 PNNs are negatively regulated by neuronal activity. Collectively, our findings demonstrate that CA2 PNN development is regulated by Mecp2 and identify a window of hippocampal plasticity that is disrupted in a mouse model of RTT.
\end{abstract}

\section{Introduction}

Rett syndrome (RTT) is a neurodevelopmental disorder caused by a loss-of-function mutation in the gene methyl-CpG-binding protein 2 (MECP2), affecting about 1 in 10,000 girls worldwide $(1,2)$. The MECP2 gene is located on the X chromosome, and $\mathrm{MeCP} 2$ functions as a transcriptional regulator, both activating and repressing various target genes $(3,4)$. A defining characteristic of RTT symptomatology is apparently normal development in the first year of life, followed by a rapid, profound regression in cognitive, motor, and social function. Some common symptoms of RTT include loss of coordination and language skills, development of stereotypic hand movements, severe autonomic dysfunction, and recurrent seizures (5-10). It is also typical for girls with RTT to exhibit autistic-like behaviors such as social avoidance $(11,12)$. Early studies of postmortem brain tissue from individuals with RTT revealed a reduced number of dendritic spines and presynaptic markers, and rodent studies revealed deficits in neurogenesis, synaptic plasticity, and experience-dependent synapse remodeling $(1,13-16)$. Although symptoms develop approximately 1 year into postnatal life in humans, abnormalities in the RTT mouse brain appear to develop prior to the presentation of symptoms (17-24). Much research has focused on understanding the initiation and progression of RTT and how MECP2 mutation may disrupt critical periods of early learning and memory.

Among the pathologies that have been observed in the RTT brain are aberrant perineuronal nets (PNNs), a specialized extracellular matrix (ECM), which typically deposit around inhibitory

Conflict of interest: The authors have declared that no conflict of interest exists. Copyright: () 2021, American Society for Clinical Investigation.

Submitted: February 12, 2020; Accepted: June 25, 2021; Published: August 16, 2021

Reference information: J Clin Invest. 2021;131(16):e137221.

https://doi.org/10.1172/JCl137221. neurons in the brain and have been implicated in several psychiatric and neurological disorders (25-29). In the normal brain, PNNs first appear during postnatal development and gradually increase until they are fully mature in adulthood (29-32). Their development is modulated by early-life experience (33-36), and mature PNNs can be altered by neuronal activity, such as seizure (37-39). PNNs have been implicated in a wide variety of functions, with recent focus on their role in limiting plasticity during critical windows of development (40-44). PNN pathology was first identified in motor cortices of postmortem human RTT tissue, where PNN-positive neurons were both more numerous and more intensely stained in layers III and V of motor cortex in comparison with control cases (27). Similarly, in a mouse model of RTT, PNNs associated with inhibitory neurons in the visual cortex were found to mature precociously and to be associated with an accelerated onset and closure of critical-period plasticity (45-47). These findings reveal a link between the loss of Mecp 2 and PNN pathology in several different brain regions and a possible link between PNN pathology and impaired critical windows of plasticity. To this end, we sought to investigate PNNs in the hippocampus of a mouse model of RTT (genetic knockout of the Mecp2 gene; Mecp2-null), a region associated with many learning and memory impairments exhibited in RTT.

Several transgenic and knockout mouse models of RTT have been generated that mimic human RTT clinical symptomatology and exhibit both neurobiological and behavioral impairments (14, $48,49)$. These mice exhibit reductions in dendritic spine number, impairments in excitatory and inhibitory transmission, aberrant neurogenesis, and disorganization of axonal fibers (18, 50-52). Although RTT typically affects young girls, Mecp2-null male mice are often used as a model of RTT, for several reasons: (a) a homozygous knockout of Mecp2, an X-linked gene, is uncommon in the Mecp2-null female (Mecp2-/) because Mecp2-null males are unable 
to breed, while the heterozygous Mecp2-null $\left(\mathrm{Mecp}^{-/ X}\right)$ results in a partial knockout and mosaic expression of Mecp2 in the brain (53); (b) heterozygous females, which we used for breeding in this study, do not present Rett-like symptomatology during early postnatal development but instead present delayed and variable phenotypic progression; (c) the onset of pathology in the Mecp2-null males occurs in early postnatal development, a delay that generally mimics the apparently normal early development and subsequent decline around 10-14 months of age that are characteristic of individuals with RTT; and (d) in general, the severity of phenotypic impairments observed in the Mecp2-null males more closely models the presentation of clinical features relative to the heterozygous female. Whether neurological dysfunction develops prior to the onset of RTT symptoms remains unclear, but several rodent studies suggest that abnormalities are present at presymptomatic ages $(19,22,54,55)$. Understanding when and how neurological dysfunction develops in the absence of Mecp2 will be critical for the ultimate goal of identifying early windows of intervention in individuals with RTT.

In this study, we aimed to determine whether PNN pathology was present in the hippocampus of a mouse model of RTT during early development. We focused specifically on hippocampal area CA2, a population of excitatory pyramidal neurons that distinctly express PNNs in the hippocampus of both mouse and human (29, 56-59). Unlike in neighboring hippocampal subregions, CA2 synapses are resistant to the induction of plasticity at synapses in the stratum radiatum (60). The function of plasticity resistance in CA2 remains to be established, although recent work points to a role for CA2 in social learning and behavior (61-65). We previously identified PNNs as a negative regulator of plasticity at CA2 synapses of acute hippocampal slices $(29,66)$. Given that MeCP2 is a known regulator of critical-period plasticity in the developing brain and that PNNs are heavily implicated in regulating critical windows of plasticity, we aimed to determine whether Mecp2 deletion resulted in PNN pathology in the developing hippocampus, particularly in area CA2 where PNNs are observed around pyramidal neurons.

\section{Results}

PNNs are increased in human RTT CA2 and develop prematurely in CA2 of a mouse model of RTT. PNNs function as at least one brake on synaptic plasticity in CA2 (29). Interestingly, previous studies have found that PNN structural complexity is increased in motor cortex of individuals with RTT and in visual cortex of Mecp2-null mice $(27,47)$. To determine whether PNNs may be altered in the human RTT hippocampus, we stained postmortem human tissue from an individual with RTT (NIH NeuroBioBank) with an antibody against the PNN component HAPLN1 link protein (58). First we identified CA2 pyramidal cells in human hippocampus with the CA2 marker RGS14 (67) (Figure 1A). We next validated that PNNs localize to human area CA2 in control tissue. We found that HAPLN1 stain was concentrated in area CA2 (Figure 1B) and that the HAPLN1 stain was darker in hippocampal tissue from the individual with RTT than in tissue from an age-matched healthy individual (Figure 1B). Higher-magnification images revealed dense localization of HAPLN1 stain to stratum pyramidale and stratum radiatum (SR) layers, with the highest intensity of staining being in the SR of the individual with RTT. Given the variability of the qual- ity of human tissue, we next sought to determine whether PNN deposition may be similarly altered in CA2 in a mouse model of RTT in which we could more precisely control staining conditions.

Clinical features of RTT typically present after the first year of life and rapidly increase in severity over the next several years (68). We therefore investigated PNN deposition in the hippocampus over the course of postnatal development in a mouse model of RTT wherein Mecp2 is deleted (Mecp2-null). Staining for mature PNNs with the marker Wisteria floribunda agglutinin (WFA) first appears in hippocampal area CA2 around postnatal day 14 (P14) in control mice and increases in intensity up to adulthood (29). We therefore quantified WFA staining in tissue from Mecp2-null animals at P14, P21, and P45 and found that staining for WFA was significantly greater in area CA2 in Mecp2-null mice compared with WT littermates at each of these ages (2-way ANOVA, Bonferroni's post hoc test, ${ }^{* *} P<0.004,{ }^{* * *} P<0.0001$, normalized to WT P45; Figure 1, $\mathrm{C}$ and $\mathrm{D})$. Interestingly, WFA staining around $\mathrm{PNN}^{+}$interneurons in area CA1 and CA3 did not differ between Mecp2-null and WT littermates $(P>0.05$; Figure 1D). We next looked at an age prior to the normal maturation of PNNs at P14 observed in WT mice to determine whether CA2 PNNs may develop precociously in RTT. We found that PNN staining was more intense at P11 in Mecp2null mice compared with WT littermates $\left({ }^{* *} P=0.0005\right.$, 2-tailed unpaired $t$ test, normalized to WT littermate; Figure 1, E and F), indicating that PNNs develop prematurely in CA2 of RTT mice.

MeCP2 is expressed in multiple cell types throughout the brain, including inhibitory interneurons, astrocytes, and microglia, which have been implicated in the behavioral deficits observed in RTT model mice (69-72). We therefore targeted the deletion of Mecp2 to CA2 pyramidal neurons to investigate the cell-autonomous effect of the loss of Mecp2 in CA2 pyramidal neurons. We crossed a line of mice expressing Cre recombinase in CA2 pyramidal neurons (Amigo2-Cre; ref. 73) with a floxed-Mecp2 mouse. This targeted deletion resulted in an increase in WFA staining in CA2 - similar to that observed in the Mecp2-null mice - and not in $\mathrm{PNN}^{+}$neurons in neighboring CA1 and CA3 regions (Supplemental Figure 1; supplemental material available online with this article; https://doi.org/10.1172/JCI137221DS1).

Potentiation at CA2 SR synapses can be induced in early postnatal development, before PNN maturation. To determine whether this premature development of PNNs had effects on synaptic plasticity, we first needed to characterize plasticity earlier than P11 in WT mice. We previously reported that excitatory responses evoked at CA2 SR synapses in P14-18 mice do not express typical long-term potentiation (LTP), and that enzymatic PNN degradation enables LTP induction (29). Because we found that PNNs are sufficient to suppress LTP at excitatory synapses in CA2, we hypothesized a relationship between the onset of PNN deposition and capacity for LTP induction. First, we validated that mature PNNs were indeed undetectable in area CA2 at P11 in WT mice using a sensitive DAB reaction (Figure 2A; see also Noguchi et al. for staining of the PNN marker aggrecan [ref. 59]). We next tested whether LTP could be induced at CA2 SR synapses at an age prior to PNN maturation $(<\mathrm{P} 14)$ in WT C57BL/6 male mice. In acutely prepared hippocampal slices, stimulating CA2 SR synapses (Figure 2B) with an LTP pairing protocol ( $3 \mathrm{~Hz}$ stimulation while depolarizing the cell to 0 $\mathrm{mV}$ ) was sufficient to induce LTP at P8-11 CA2 SR synapses from 

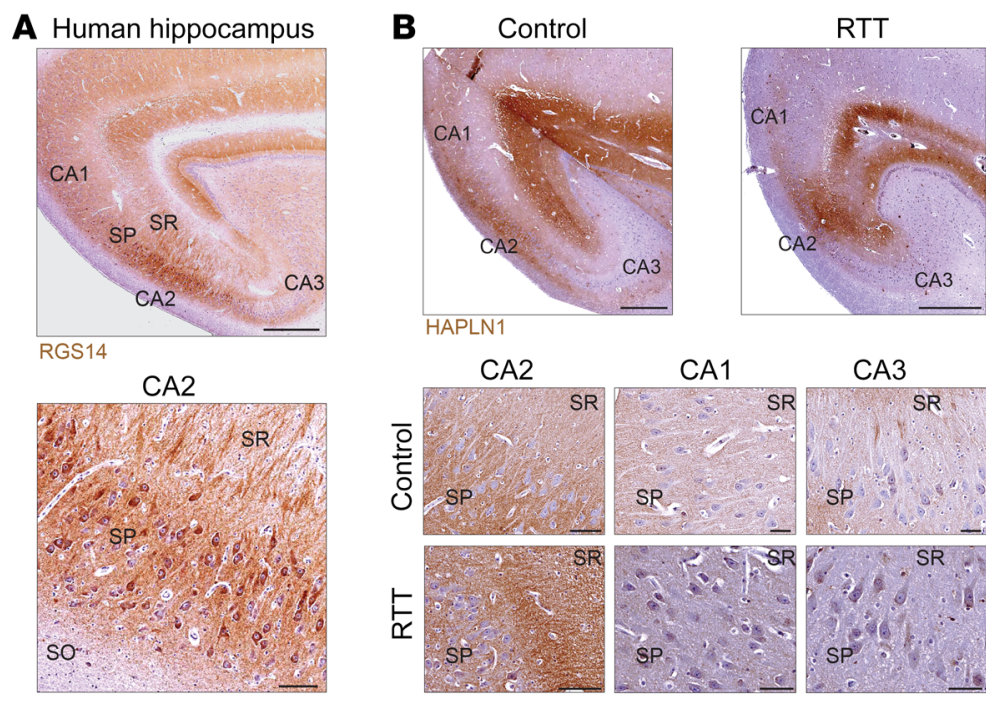

CA3

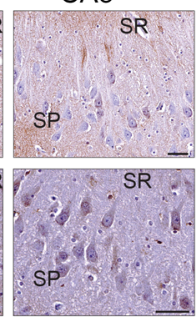

C Mouse hippocampus WT P14

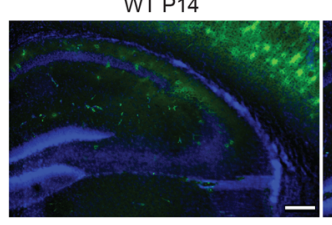

WT P21

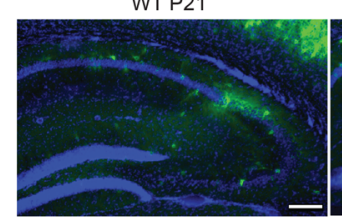

WT P45
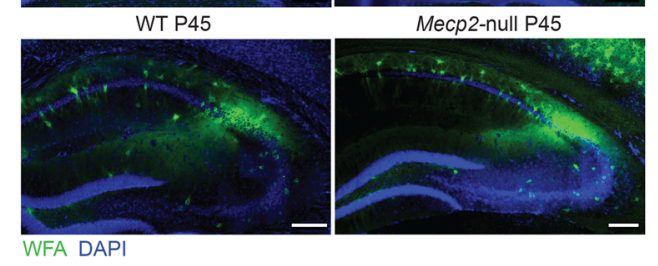

E

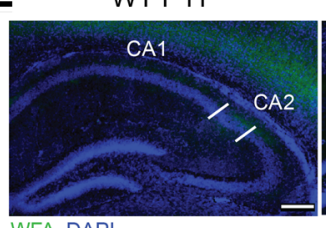

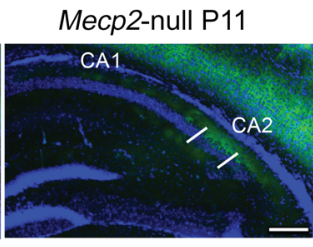

D

Mecp2-null P14

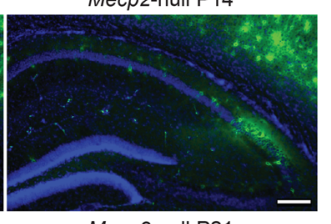

Mecp2-null P21

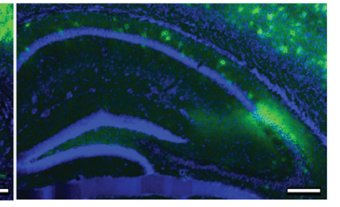

Mecp2-null P45

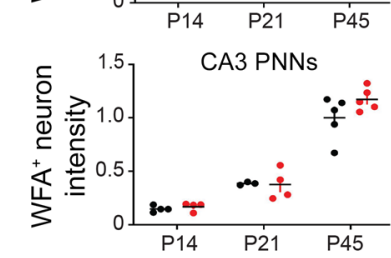

$\mathbf{F}$

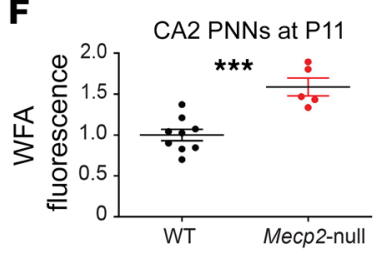

Figure 1. PNNs are increased in human RTT CA2 and develop prematurely in CA2 of a mouse model of RTT. (A) Staining for the CA2-enriched protein RCS14 labels CA2 pyramidal neurons in control human hippocampal tissue in the stratum pyramidale (SP) and stratum radiatum (SR), but less in the stratum oriens $(\mathrm{SO})$. Scale bars: $1 \mathrm{~mm}$ (top), $100 \mu \mathrm{m}$ (bottom). (B) HAPLN1, a PNN link protein, is localized to area CA2 in a healthy human hippocampus (relative to background: CA1, 0.57; CA2, 1.67; CA3, 0.082). HAPLN1 appears greater in hippocampal tissue from an age- and sex-matched individual with RTT (relative to background: CA1, 1.06; CA2, 2.87; CA3, 0.52). Scale bars: $1 \mathrm{~mm}$ (top), $100 \mu \mathrm{m}$ (bottom). (C) Left: Staining for the PNN marker WFA (green) is greater in Mecp2null CA2 compared with WT littermates throughout early postnatal development. Scale bars: $200 \mu \mathrm{m}$. (D) Normalized WFA fluorescence intensity was significantly greater in Mecp2-null CA2 ( $n=4,4,5$ for ages P14, P21, P45, respectively) compared with WT littermates $(n=4,3,5$ for ages P14, P21, P45, respectively); ${ }^{* *} P=0.004,{ }^{* * * *} P<$ 0.0001 , 2-way ANOVA, Bonferroni's post hoc test, significant main effects of age $F_{219}=194.8$, condition $F_{119}=110.5$, and interaction $F_{2,19}=4.913$. Indicated are means \pm SEM In addition, WFA staining surrounding $\mathrm{PNN}^{+}$neurons in CA1 and CA3 did not differ between Mecp2-null males and WT littermates; $P>0.05$, 2-way ANOVA. Indicated are means \pm SEM. (E) Staining intensity for WFA (green) is greater in area CA2 of Mecp2-null at P11 compared with WT littermate. The CA2 pyramidal neuron borders with areas $C A 1$ and $C A 3$ are represented with white lines. Scale bars: $200 \mu \mathrm{m}$. (F) Normalized WFA fluorescence intensity was significantly greater in CA2 of P11 of Mecp2-null; ${ }^{* *} P=0.0005,2$-tailed unpaired $t$ test $(n=9$ and 5 , WT littermate and Mecp2-null, respectively). Indicated are means \pm SEM. Fluorescence intensity was amplified by camera exposure settings relative to exposure settings in C for quantification purposes.
WT mice $(1.51 \pm 0.2$ baseline, $n=8)$ but not at P14-18 (0.87 \pm 0.09 baseline, $n=10)\left({ }^{*} P=0.0104,2\right.$-tailed unpaired $t$ test at $25-30$ minutes; Figure 2C).

To further investigate electrophysiological differences in CA2 neurons in WT mice at P8-11 versus P14-18, we examined intrinsic cell properties and synaptic responses. Resting membrane potentials and action potential (AP) thresholds did not differ in a way that could explain the lack of LTP at P14-18; however, as expected given the growth of dendrites during this age range, capacitance was lower at P8-11 and input resistance was higher at P8-11 in comparison with P14-18 CA2 neurons $\left({ }^{*} P=0.0041\right.$ and ${ }^{\star} P=0.00032$, respectively, 2-tailed unpaired $t$ test; Supplemental Table 1$)$. These findings, not surprisingly, suggest that at P8-11 CA2 neurons may be smaller and perhaps more easily depolarized during the pairing protocol. We next tested baseline synaptic transmission in CA2 and found that synaptic responses were larger at P8-11 compared with P14-18 CA2 neurons at the highest current stimulation intensities $\left({ }^{* *} P=0.0026\right.$ at $320 \mu \mathrm{A}$ of stimulation, $n=9$ and 8 , respectively, 2-way ANOVA, Bonferroni's post hoc test; Figure 2D). However, synaptic responses did not differ at the stimulation intensities used in our LTP induction protocol (15-30 $\mu \mathrm{A})$ (Figure 2D, inset). Pairedpulse facilitation (PPF) did not differ between age groups (2-tailed unpaired $t$ test, $P>0.05, n=14$ for P8- 11 and $n=7$ for P14-18; Figure $2 \mathrm{E}$ ), and $\mathrm{AP}$ firing frequency of CA2 neurons was greater at P14-18 compared with P8-11 over a range of injected currents $\left({ }^{*} P\right.$ $=0.0185$ at $180 \mathrm{pA}$ of injected current, $n=20$ and 13 , respectively, 
A
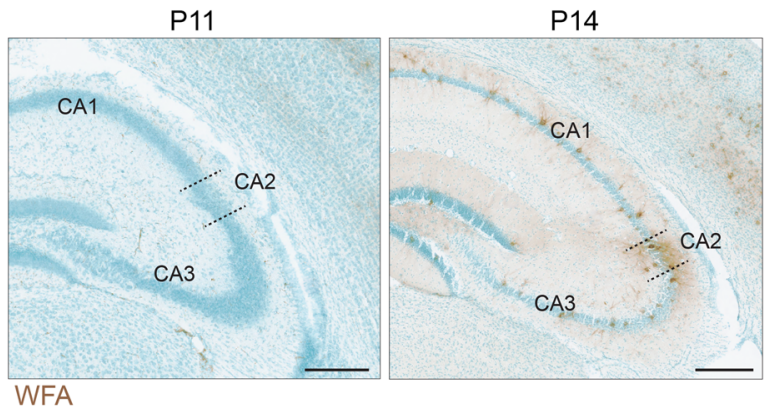

CA2 P8-11

C
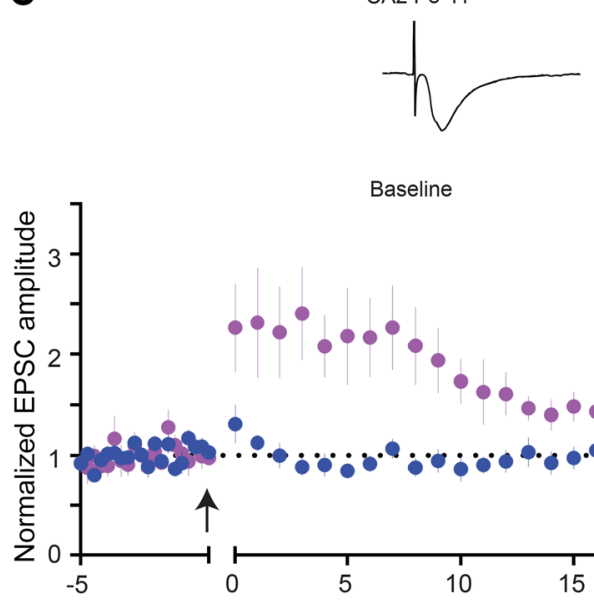

Baseline
P14

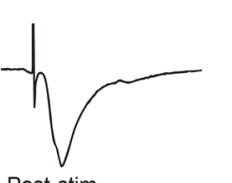

B
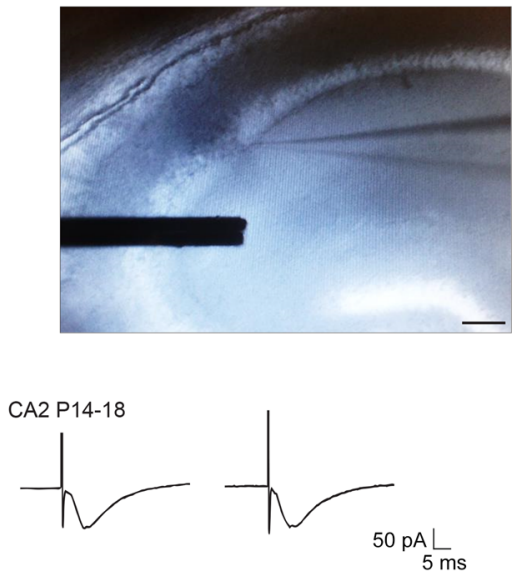

Baseline
Post-stim

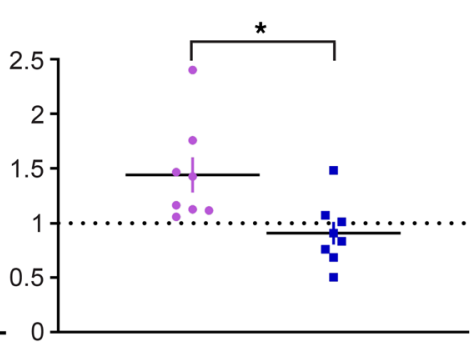

D

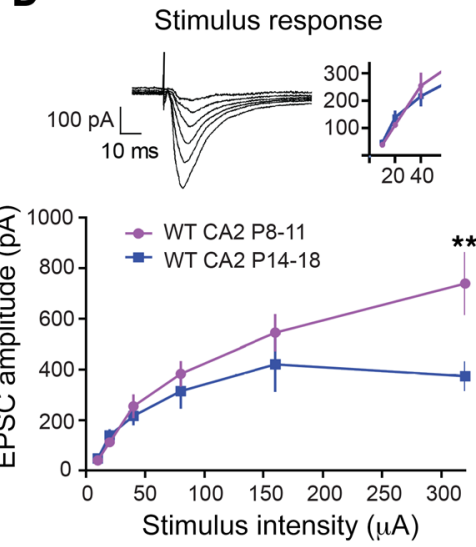

E

Paired-pulse facilitation
F Action potential firing frequency

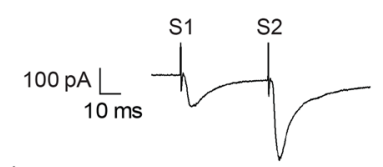
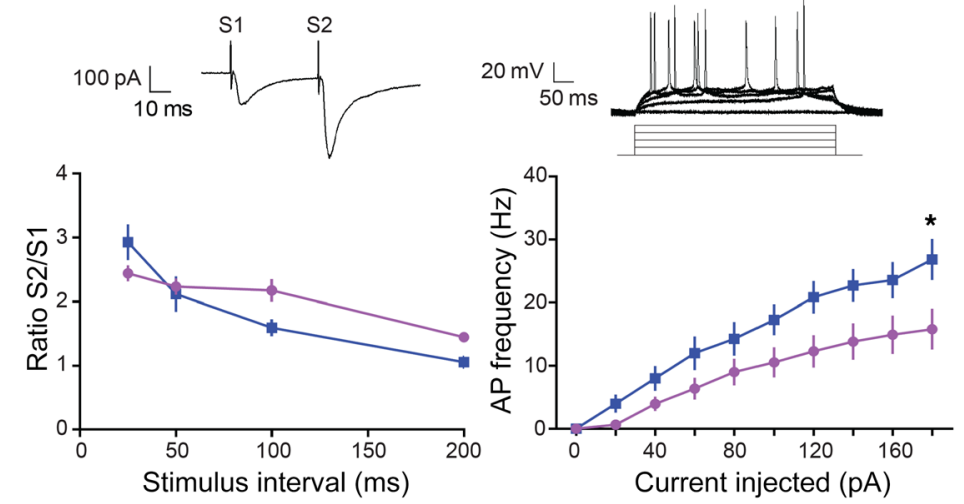

Figure 2. Potentiation in CA2 can be induced before PNN maturation. (A) Staining for PNNs as defined by WFA is morphologically mature in CA2 at P14 (right) but not at P11 (left). Scale bars: $250 \mu \mathrm{m}$. (B) Representative image of a 300- $\mu \mathrm{m}$-thick hippocampal slice showing the placement of a recording electrode in CA2 and a stimulating electrode in CA3 stratum radiatum. Scale bar: $100 \mu \mathrm{m}$. (C) In slices from WT mice, excitatory postsynaptic current (EPSC) amplitudes recorded in CA2 neurons increased in response to an LTP pairing protocol (270 pulses at $3 \mathrm{~Hz}$ paired with postsynaptic depolarization at time 0 ) at P8-11, but not in slices harvested at P14-18 ( $n=8$ for P14-18, in blue; $n=9$ for P8-11, in purple). Top: Representative traces of EPSCs from CA2 neurons before and 25 minutes after the pairing protocol. Arrow indicates time of the pairing. Indicated are means \pm SEM, normalized to baseline. Right: Normalized mean response amplitudes averaged over 25-30 minutes of recovery after pairing; ${ }^{*} P=0.0104,2$-tailed unpaired $t$ test. (D) EPSC amplitudes in response to indicated stimulation intensities were larger at P11 compared with P14; ${ }^{* *} P=0.0026$ at 320 pA, 2-way ANOVA, Bonferroni's post hoc test ( $n=9$ and 8, P8-11 and P14-18, respectively). Top: Representative traces from a CA2 neuron at P10 in response to increasing stimulation intensities. Inset displays responses at lower stimulation intensities, the range at which LTP experiments were performed. (E) Paired-pulse ratio was unchanged between P8-11 and P14-18 at CA2 synapses; $P>0.05$. Top: Representative trace from WT CA2 P11 neuron in response to a 50-millisecond stimulus interval (S1, peak of first stimulus response; S2, peak of second stimulus response). (F) Action potential firing frequency of P14-18 CA2 neurons was greater compared with that at P8-11 at 180 pA of injected current; ${ }^{*} P=0.0185$, 2-way ANOVA, Bonferroni's post hoc test ( $n=13$ and 20, P8-11 and P14-18, respectively). Top: Representative traces of action potentials recorded in response to current injections from 0 to 180 pA in WT P11. 
2-way ANOVA, Bonferroni's post hoc test; Figure 2F). Note that the stimulus-response and PPF experiments were performed without GABA blockers because of epileptiform activity during recordings at P8-11. Because we found that PNNs developed prematurely in the Mecp2-null mice, we next investigated how the loss of Mecp2, a known regulator of critical-period plasticity, impacts this apparently novel window of CA2 synaptic plasticity.

Synaptic potentiation is prematurely restricted at CA2 SR synapses in a mouse model of RTT. We next tested whether the precocious development of PNNs alters LTP induction in Mecp2-null CA2. First, we replicated the finding that plasticity occurs in CA2 at P8-11 in a separate cohort of male WT littermates (Figure 3A). Consistent with the finding that global loss of Mecp2 accelerates the closure of critical-period plasticity in the visual cortex $(45,47$, 74), we found that potentiation in CA2 was prematurely restricted at P8-11 SR synapses in Mecp2-null compared with WT littermates (1.10 \pm 0.2 baseline vs. $1.72 \pm 0.1$ baseline in WT at 18-20 minutes, $n=13$ and 14, respectively, ${ }^{* *} P=0.0031$, 2-tailed unpaired $t$ test; Figure $3 \mathrm{~A})$. Baseline synaptic transmission, as assessed with a stimulus-response curve, was not different at P8-11 CA2 synapses of Mecp2-null compared with WT littermates $(P>0.05$ at 320 pA current stimulation, 2-way ANOVA repeated measures, Šidák's multiple-comparison test; Figure 3B). In addition, neither PPF nor AP firing frequency differed significantly between Mecp2-null and WT littermates at P8-11 ( $P>0.05$, 2-way ANOVA; Figure 3, C and $D)$. Intrinsic properties did not differ in a way that could explain the premature restriction of plasticity in CA2 $(P>0.05$, unpaired $t$ test; Supplemental Table 1). Although we did not perform these experiments in the presence of GABA blockers because of epileptiform activity, we did perform stimulus-response and PPF experiments at P14-18 in the presence of the $\mathrm{GABA}_{\mathrm{A}}$ receptor blocker bicuculline, and found no overall difference between Mecp2-null and WT littermates in excitatory response size or PPF $(P>0.05$, 2-way ANOVA repeated measures, Šidák's multiple-comparison test; Supplemental Figure 2).

PNN degradation restores plasticity at CA2 Mecp2-null synapses. Because we found an increase in both PNNs and another plasticity-restricting protein, RGS14, in RTT CA2 at P10 (Supplemental Figure 3A), we sought to determine whether the precocious increase in PNNs alone was sufficient to explain the premature restriction of LTP in RTT CA2. In this experiment, we tested whether degrading PNNs with the exogenous enzyme chondroitinase $\mathrm{ABC}(\mathrm{ChABC})$ in acutely prepared hippocampal slices was sufficient to enable LTP induction in RTT CA2 at P8-11. We previously reported that degrading PNNs with $0.05 \mathrm{U} / \mathrm{mL} \mathrm{ChABC}$ for $\geq 2$ hours is sufficient to degrade PNNs in CA2 in slices (see Figure 4, and Methods in ref. 29). Following the same protocol, we found that degradation of PNNs in P8-11 Mecp2-null hippocampal slices "restored" the capacity for potentiation in CA2 $(1.57 \pm 0.2$ baseline vs. $0.90 \pm 0.1$ baseline in untreated Mecp2-null at 18-20 minutes, $n=14$ and 10, respectively, ${ }^{*} P=0.035$, 2-tailed unpaired $t$ test; Figure $3 \mathrm{E}$ ). Intrinsic properties did not change in a way that would explain the restoration of LTP at CA2 synapses with ChABC treatment (Supplemental Table 1). Taken together, these data demonstrate that degradation of the aberrantly increased PNNs in RTT CA2 appears sufficient to restore synaptic plasticity in CA2 at young postnatal ages. To better understand what might be driving the precocious increase of PNNs in RTT CA2, and because seizure activity is comorbid with RTT, we next investigated the role of aberrant neuronal activity as a potential mechanism upregulating PNNs in CA2 $(38,75)$.

CA2 PNNs are inversely regulated by neuronal activity in vivo. PNN development is dependent on early-life experience and neuronal activity, shown in several sensory brain regions and across several species $(32,33,35,75-77)$. For example, visual deprivation from birth delays the deposition of PNNs and attenuates overall expression levels in the visual cortex (34). Accordingly, we hypothesized that a precocious upregulation of PNNs in RTT CA2 is driven by increased hippocampal activity (78). To investigate this, we directly manipulated CA2 neuronal activity using a chemogenetic approach, designer receptors exclusively activated by designer drugs (DREADDs). To target CA2 pyramidal neurons, we infused an AAV vector encoding Cre-dependent Gq-coupled (excitatory) or Gi-coupled (inhibitory) DREADDs into CA2 of adult mice expressing Cre recombinase in CA2 neurons (79). In a previous study from our laboratory, we found that clozapine $\mathrm{N}$-oxide $(\mathrm{CNO})$ increased the firing rate of CA2/proximal CA1 pyramidal neurons in mice expressing excitatory DREADDs (hM3Dq) in CA2 (79). Similarly, CNO decreased CA2 responses in mice expressing the inhibitory DREADD (hM4Di), with effects lasting up to 24 hours after DREADD activation (79). In this study, we either chronically increased or chronically decreased CA2 activity for 5 days and quantified WFA fluorescence in the hippocampus (Figure 4A). Contrary to our hypothesis, we found that increased neuronal activity in the Gq DREADD-expressing animals significantly decreased WFA staining in CA2 in comparison with Crecontrols ( ${ }^{*} P=0.0111$, unpaired 2-tailed $t$ test; Figure 4, B and $\mathrm{C}$ ). Conversely, we found the opposite in the Gi DREADD-expressing animals: WFA staining was significantly increased in CA2 in comparison with Cre- controls $\left({ }^{*} P=0.0179\right.$, unpaired 2-tailed $t$ test). We also found that WFA staining in inhibitory neurons in areas CA1 and CA3, which did not express the DREADDs, was unchanged in Gi DREADD- or Gq DREADD-expressing mice $(P>$ 0.05; Supplemental Figure 3B). We did, however, observe a significant increase in WFA fluorescence in overlying primary somatosensory cortex in Gq DREADD mice, suggesting that altered CA2 activity has extrahippocampal effects $\left({ }^{* *} P=0.0012\right.$, 1-way ANOVA, Tukey's post hoc test; Supplemental Figure 3B). Overall, these data demonstrate that PNNs in CA2 are cell-autonomously regulated by aberrant neuronal activity and suggest that PNNs in CA2 are negatively regulated by activity. Notably, neuronal activity in these experiments was manipulated in adulthood, leaving open the possibility that aberrant changes in activity at even younger ages may still explain abnormal PNN maturation.

In order to investigate the effects of aberrant activity on PNNs earlier in postnatal development, we next studied the effects of pathological activity using a mouse model of epilepsy. The development of seizures in childhood and persistent epilepsy is a common and devastating comorbidity of RTT $(28,80,81)$. Previous studies have shown that PNNs are altered by seizure activity in humans and rodents $(25,28,37,38,81)$, but a direct relationship between PNNs and abnormal neuronal activity remains unclear. Here, we quantified WFA fluorescence in the hippocampus of a mouse model of epilepsy, the Kv1.1-null mouse, a genetic knock- 

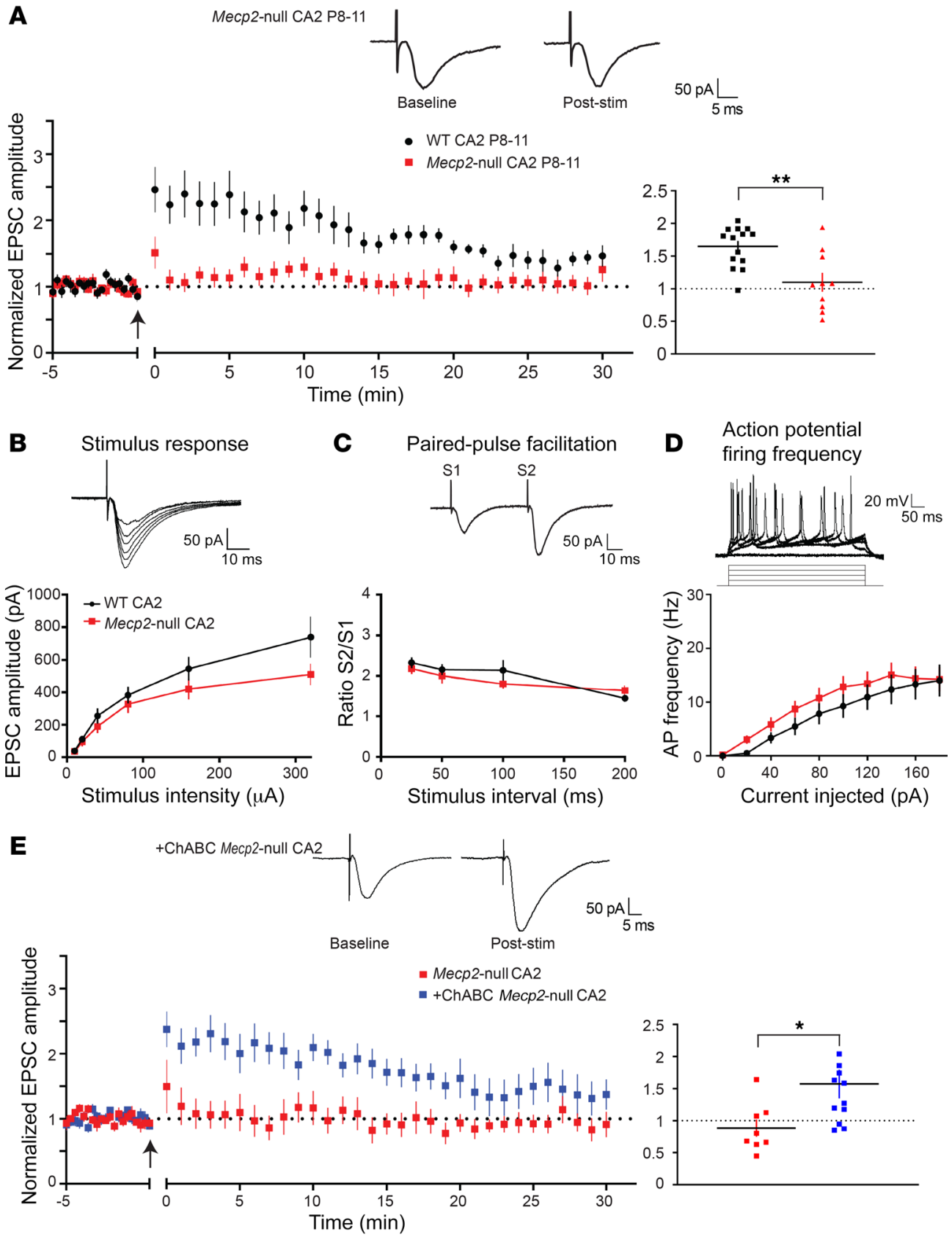

Figure 3. Potentiation is prematurely restricted in CA2 of a mouse model of RTT via PNNs. (A) Potentiation failed to be induced at CA2 stratum radiatum synapses at ages P8-11 in acute hippocampal slices from Mecp2null mice (red) compared with WT (black). Top: Representative EPSCs from Mecp2-null P8-11 CA2 neurons at baseline and 20 minutes after the LTP pairing protocol (post-stimulation). Right: Normalized mean response amplitudes 18-20 minutes after pairing; ${ }^{* *} P=0.0031$, 2-tailed unpaired $t$ test $(n=14$ and 13 , WT littermate and Mecp2-null, respectively). (B) EPSC amplitudes in response to indicated stimulation current intensity were not different in Mecp2-null CA2 neurons compared with WT littermate at P8-11; main effect of genotype $P=0.2738$, 2-way ANOVA, repeated-measures analysis, Šidák's post hoc test ( $n=9$ and 5, WT littermate and Mecp2-null, respectively). Top: Representative traces from a Mecp2-null CA2 neuron at P8 in response to increasing stimulation intensities. (C) Paired-pulse ratio did not differ between Mecp2-null and WT CA2 synapses (S1, peak of first stimulus response; S2, peak of second stimulus response). Top: Representative traces using a 50-millisecond stimulus interval from a P11 Mecp2-null CA2 neuron; average of 4 responses. (D) Action potential firing frequency of Mecp2-null CA2 neurons did not differ in comparison with WT; $P>0.05,2$-way ANOVA ( $n=12$ and 18 , WT and Mecp2-null, respectively). Top: Representative traces of action potentials from 0 to $180 \mathrm{pA}$ current injection in a P8 Mecp2-null CA2 cell. (E) Degradation of PNNs by incubation of slices in vitro with chondroitinase $A B C$ (ChABC) for 2 hours resulted in potentiation being restored. Top: Representative EPSCs from CA2 +ChABC Mecp2-null P8 neurons at baseline and 20 minutes after LTP pairing (post-stimulation). Right: Normalized mean response amplitudes at 18-20 minutes of recovery; ${ }^{*} P=0.035$, 2-tailed unpaired $t$ test $(n=14$ and 10, Mecp2-null and +ChABC Mecp2-null, respectively). out of the potassium channel Kv1.1 (Kcna1) (82). Kv1.1-null mice exhibit spontaneous and recurrent seizures beginning at P21 (82). During a 1-hour recording of a Kv1.1-null mouse (P40), we recorded 3 episodes of ictal activity (Figure 4D), each lasting 25-30 seconds, followed by non-ictal activity for approximately 15-20 minutes, indicating that these mice display frequent, recurrent seizure activity. We found that PNN staining was decreased in CA2 by P45-60 in the Kv1.1-null mice but was unchanged at younger ages, after the initial onset of seizures (P22 and P28; Figure 4, E and F). Interestingly, we found the opposite effect in the dentate gyrus, where PNNs were dramatically increased at P45-60, similar to what we recently found in a different model of epilepsy in Angelman syndrome model mice (25). Overall, PNNs appear to be regulated by aberrant activity in vivo in CA2; however, contrary to our predictions, we found that CA2 PNNs were negatively regulated by activity. Because activity has been reported to be abnormally increased in Mecp2-null mice (78), our findings do not readily explain the upregulation of PNNs. However, these data may be interpreted to indicate hypoexcitability in CA2. To gain further insight into the early PNN upregulation in postnatal development of RTT, we next examined potential molecular mechanisms in these Mecp2-null mice.

Loss of Mecp2 alters the molecular profile of the developing hippocampus. MeCP2 plays an important role in activity-dependent transcriptional regulation in postnatal development, and loss of MeCP2 results in misregulation of numerous neuronal transcripts important for activity-dependent plasticity $(4,83-$ $85)$. We sought to identify molecular changes that may explain the dysregulation of PNNs and 
A

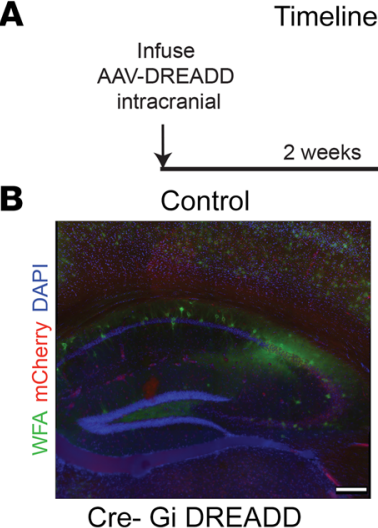

Cre- Gi DREADD

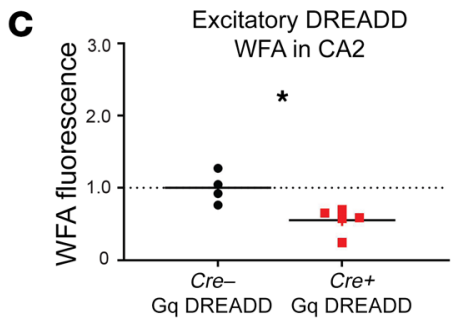

D Mouse model of epilepsy: Kv1.1-null
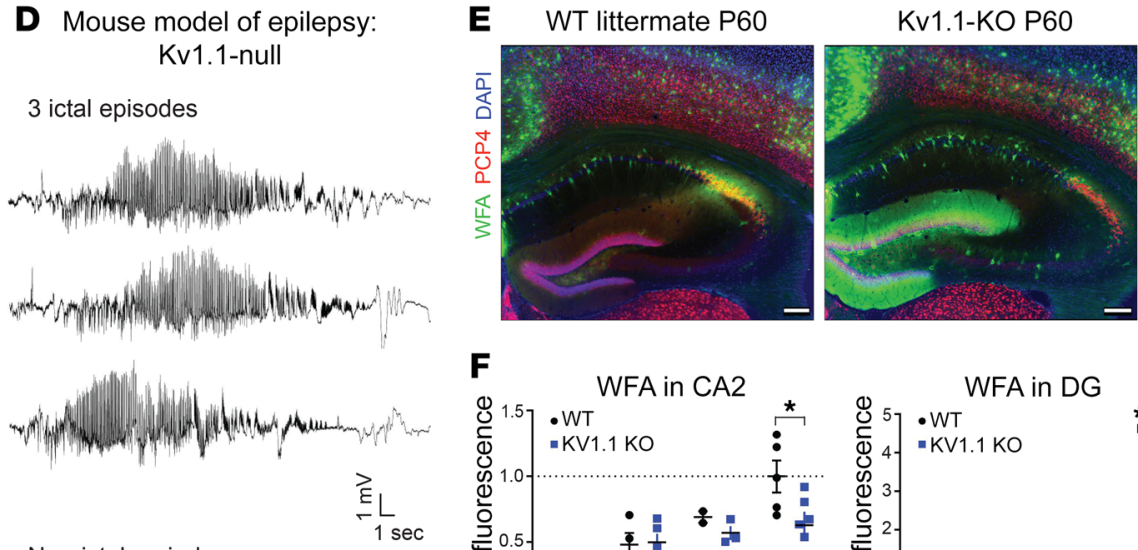

Non-ictal period
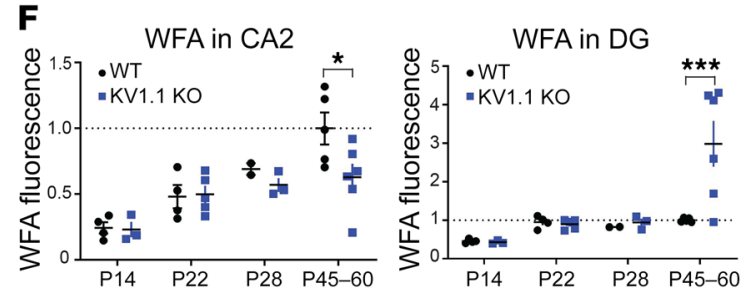

Figure 4. CA2 PNNs are inversely regulated by neuronal activity in vivo. (A) Experimental timeline indicating the infusion of the DREADD-AAV into the hippocampus of Amigo2-iCreERT2+ and Amigo2iCreERT2- mice. Two weeks later, tamoxifen injections were given for 7 days to induce selective expression in CA2 neurons. Two weeks after the last tamoxifen injection, the DREADD ligand CNO was given twice daily for 5 days ( $1 \mathrm{mg} / \mathrm{kg}$ for Gq DREADD, $5 \mathrm{mg} / \mathrm{kg}$ for Gi DREADD, s.c.). (B) Immunofluorescence shows mCherry-Gq DREADD (red) only in CA2 neurons. Representative images of WFA fluorescence intensity (green) in the hippocampus after 5 days of CNO treatment. Scale bars: $200 \mu \mathrm{m}$. (C) Quantification of WFA staining in CA2 showed a significant reduction in Gq DREADD-expressing animals compared with Cre-controls ( ${ }^{*} P=0.011$, unpaired 2-tailed $t$ test; $n=4$ and 5 for Cre- and Cre+, respectively) and increase in CA2 of Gi DREADD-expressing animals compared with Cre- controls $\left({ }^{*} P=0.0179\right.$, unpaired 2-tailed $t$ test; $n=6$ for both Cre- and Cre+ groups). (D) Spontaneous electrographic seizures were detected in vivo in the hippocampus of a mouse model of epilepsy, the Kv1.1-null mouse. Examples of electrographic seizure activity detected by LFP recordings from the hippocampus during a 1-hour recording session in a P40 Kv1.1-null male mouse are shown. A period of non-ictal activity during the same recording session is shown for comparison. (E) Staining for PNN marker WFA (green) is reduced in CA2 of Kv1.1-null mice compared with WT littermates at P60. CA2 pyramidal neurons are identified with the CA2 marker PCP4 (red). Scale bars: $200 \mu \mathrm{m}$. (F) Normalized WFA fluorescence intensity was decreased at P45-60 in CA2 compared with WT; ${ }^{*} P=0.033$ ( $n=4,4,3$, and 5 for ages P14, P22, P28, and P45, respectively). In contrast, WFA fluorescence intensity was significantly greater in the dentate gyrus (DC) of Kv1.1-null mice compared with WT littermates at P45-60; ${ }^{* *} P=0.0002$, Bonferroni's post hoc test for pairwise comparison after 2-way ANOVA. Indicated are means \pm SEM normalized to P45 WT group. plasticity in RTT CA2. We compared gene expression profiles by age, P10 versus $\mathrm{P} 18$, and by genotype, WT versus Mecp2-null, using a custom-designed code set representing an array of genes specific to the different CA subregions and others of interest (NanoString, https://www.nanostring.com) (86). At P10, when plasticity is prematurely restricted at CA2 SR synapses in the Mecp2-null hippocampus, the CA2-enriched genes Pcp4 and Spink8 were significantly decreased in RTT compared with WT littermates (unadjusted $P=$ 0.00730 and 0.0487 , respectively; Figure 5A). The inhibitory marker Gad1, which encodes an enzyme important for the production of GABA, was also significantly decreased at P10 in Mecp2null compared with WT (unadjusted $P$ $=0.0332$ ), indicating a possible loss of inhibitory transmission in RTT hippocampus at P10. Expression of several genes was increased at P10 in Mecp2null hippocampus compared with WT, including the CA2-enriched gene Fgf2 (unadjusted $P=0.0101$ ), which encodes a protein that has been implicated in the normal development of the hippocampus and relies on ECM binding for growth factor signaling (87-90). Notably, Fgf2 is one of the few genes that were significantly increased at both P10 and P18 (adjusted $P=0.0146$ ). Interestingly, mRNAs for the CA2 plasticity-regulating genes Rgs14 and Acan (aggrecan) were not increased despite the increase in protein levels at P11 (Supplemental Figure 3). This difference may suggest a role for MeCP2 in regulating translation of these transcripts. Finally, an accelerated maturation of parvalbumin-positive $\left(\mathrm{PV}^{+}\right)$inhibitory neurons reportedly plays an important role in the upregulation of PNNs in RTT visual cortex and the regulation of critical-period plasticity $(45,47)$. However, we report here no significant difference in expression of the gene encoding PV (Parv), but note that the pattern of fold-change differences comparing Mecp2-null and WT flipped with age; Parv was lower in Mecp2-null hippocampus at P10 and was higher in Mecp2-null hippocampus at P18 $(P>0.05)$. We also found that PV staining was decreased in the $M e c p 2^{-/ Y}$ mice compared with controls at P14 

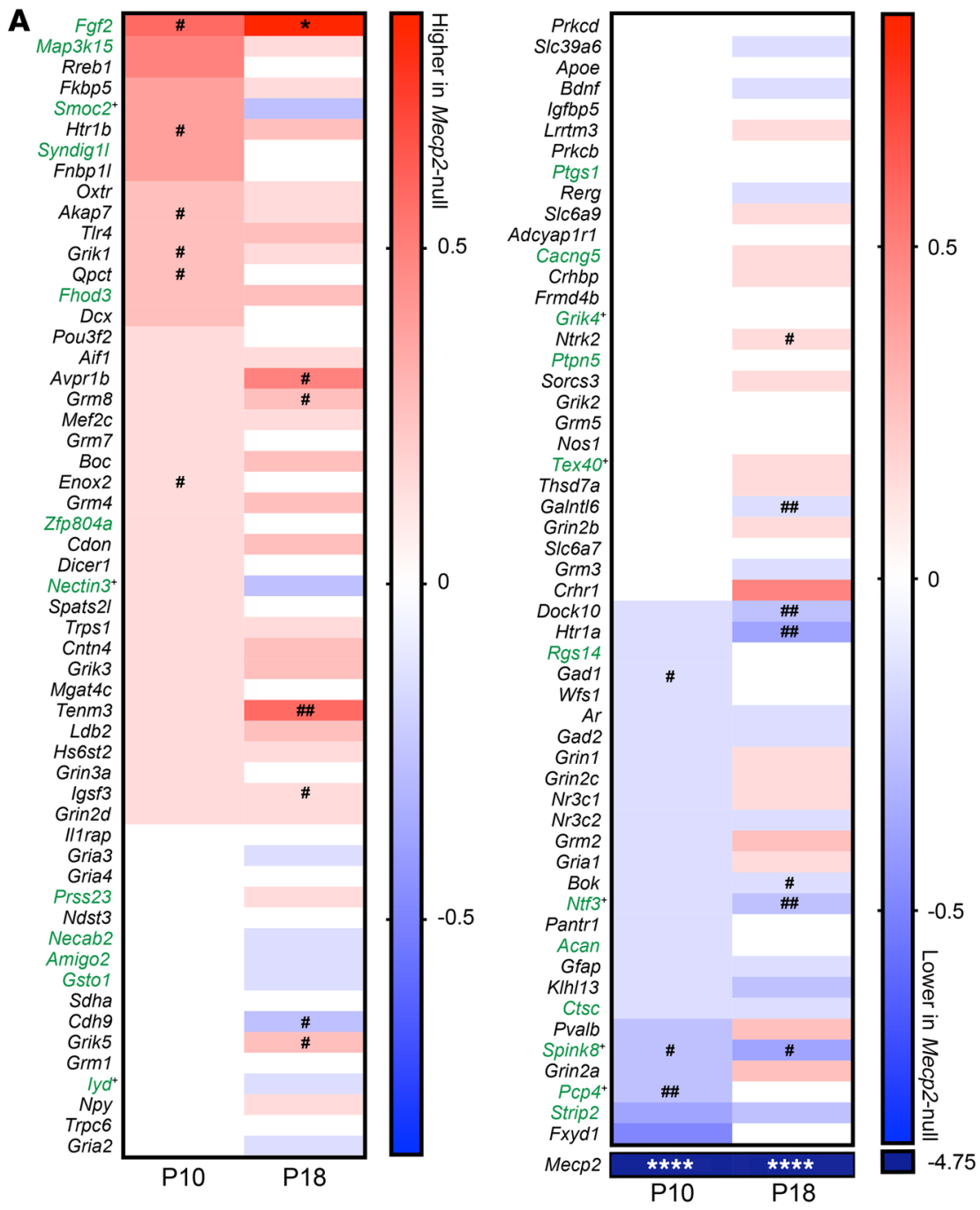

B MMP-9 protein

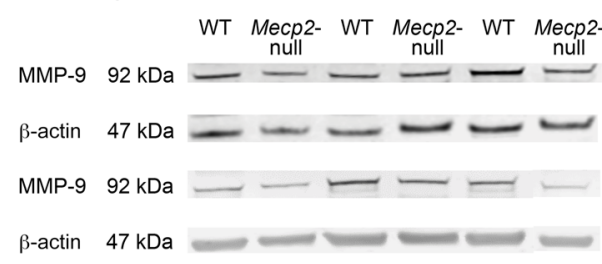

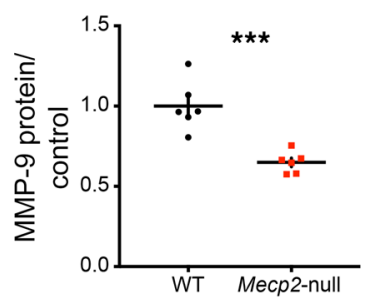

Figure 5. Loss of Mecp2 alters the molecular profile of the developing hippocampus. (A) Given the premature loss of plasticity in Mecp2-null P8-11 mice, we examined gene expression differences at P10 and P18 in Mecp2-null compared with WT littermate control. Of the CA2-enriched genes (green font), Pcp4 and Spink8 were significantly lower in Mecp2-null compared with WT littermates (both unadjusted $P$ value $=0.00730$ and $P=0.0487$, respectively). The CA2-enriched gene Fgf2 was significantly higher at both P10 (unadjusted $P=0.0101$ ) and P18 (adjusted $P=0.0146)$. The inhibitory marker Gad1 was also significantly lower at P10 in Mecp2-null compared with WT (unadjusted $P=0.0332$ ). Neither Rgs14 nor Acan (aggrecan) increased despite the increase in protein levels at P11 (Supplemental Figure 3A). We report no significant difference in the gene encoding PV (Parv), but note that the pattern of fold-change differences comparing Mecp2-null and WT depends on age. Genes with an adjusted $P$ value less than 0.05 are designated with an asterisk, and genes with an unadjusted $P$ value less than 0.05 are designated with “\#”. Differences are presented as $\log _{2}$ fold change compared with WT littermates. (B) The endogenous ECM-degrading enzyme matrix metalloproteinase 9 (MMP-9) is significantly decreased in a hippocampal lysate of Mecp2-null males compared with WT littermates $(\mathrm{P} 45-60) ;{ }^{* *} P=0.0005$, unpaired 2-tailed $t$ test ( $n=6$ for P37-42 for Mecp2-null males and for WT littermates). Right: MMP-9 band intensity from Mecp2-null males was normalized to $\beta$-actin band intensity and divided by the average of the values from WT littermates. See complete unedited blots in the supplemental material.
( $n=4, P=0.042$; data not shown), suggesting that any accelerated maturation of PV circuitry may not be occurring until after the second postnatal week in the RTT hippocampus.

Lastly, we sought to investigate the role of an important molecular regulator of PNNs, ECM-degrading proteases, which were not in our NanoString code set. PNNs are dynamically regulated by endogenous ECM-degrading enzymes, such as the matrix metalloproteinases (MMPs). Interestingly, MMP-9 has emerged as a critical regulator of neural circuit and plasticity development during early postnatal development (91-93). MMP-9 expression is dysregulated in a number of neurological disorders, such as schizophrenia and fragile $\mathrm{X}$ syndrome, which are also characterized by social and cognitive deficits (91, 93-96). We hypothesized that an upregulation of PNNs in RTT CA2 may be explained by a loss of the ECM-degrading enzyme MMP-9. Indeed, we found that MMP-9 protein was significantly decreased in Mecp2-null hippocampus in comparison with WT littermates (P50-60; Figure $5 \mathrm{~B})$. Taken together, these results suggest that decreased MMP-9 may be one possible mechanism by which PNNs are increased in CA2. We also found that several inhibitory neuron transcripts were decreased at P10 in RTT hippocampus, suggesting that enhanced inhibition is an unlikely candidate to explain the upregulation of PNNs and the accelerated loss of CA2 plasticity. Finally, we identified aberrant expression of several CA2enriched genes in the RTT hippocampus that may also be of interest for future study, such as Fgf2, a growth factor that relies on ECM binding for signaling and plays a role in hippocampal development and learning. 


\section{Discussion}

A major challenge in understanding the pathogenesis of RTT is identifying key molecular targets and determining how and when they cause synaptic dysfunction. Abnormalities in the deposition of PNNs, a specialized ECM in the brain, were discovered in the motor cortex of postmortem individuals with RTT and in a mouse model of RTT $(27,45,97)$. In this study, we report on the aberrant expression of PNNs in hippocampal area CA2 in RTT. First, we demonstrate what appears to be an increase in PNNs in an individual with RTT compared with an age- and sex-matched healthy individual, noting the limitations of data interpretations from only 2 individuals. Consistent with this finding was that we similarly found an increase and a precocious maturation of PNNs in CA2 of an RTT model mouse. Because PNNs function to limit synaptic plasticity in CA2 (29), we investigated how the accelerated maturation of PNNs may alter CA2 plasticity. We identified a period of plasticity at CA2 synapses, prior to the maturation of PNNs, and identified PNNs as a causal mechanism underlying the premature closure of CA2 plasticity in the RTT mouse. Together these data demonstrate that the timing of plasticity restriction in CA2 maps onto the development of PNNs. We explored mechanisms to explain the aberrant upregulation of PNNs and conclude that the sustained upregulation of PNNs in RTT could be explained by the downregulation of the endogenous PNN-degrading protease MMP-9 in the RTT hippocampus and not by an accelerated maturation of inhibitory circuitry or by increases in neuronal activity. Importantly, we found that the enzymatic degradation of PNNs was sufficient to rescue the plasticity deficit in RTT CA2, identifying a potential therapeutic approach to rescue the functional consequences of $\mathrm{PNN}$ overexpression in disease.

In this study, we used male hemizygous Mecp2-deficient mice. Although heterozygous female $M e c p 2^{+/-}$mice may be a more clinically relevant model of RTT, given that most affected individuals are girls, the male $M e c p 2^{-/ Y}$ mice presents several advantages (discussed in ref. 53), particularly because heterozygous females add the experimental challenges of delayed and variable phenotypic progression and cellular mosaicism due to X-linked inactivation (98-100). In addition, female heterozygous mice are asymptomatic for the first 4 months of life, in contrast to hemizygous male mice, which exhibit overt phenotypes as early as 3 weeks of age. Male and female mice, however, do share a subset of phenotypes, such as reduced dendritic complexity, spine density, and soma size in cortical regions $(17,52,101-103)$. Moreover, several behavioral phenotypes, such as disrupted contextual fear learning, anxiety-like behavior, inability to complete hippocampusdependent tasks, and social deficits, depend on the behavioral test, mouse model, and age tested $(99,104-106)$. Thus, in many ways, although the male mice are more severely affected than females, they do provide certain advantages for study.

PNNs and a critical period for synaptic plasticity in CA2. PNN maturation closely tracks the closing of critical windows of plasticity in several different brain regions and is functionally implicated in inhibiting plasticity in the developing brain (30). For example, in the mouse visual system, PNNs first appear around inhibitory neurons after eye opening (P15) and are fully matured by $\mathrm{P} 30$, tracking the closure of critical-period plasticity (45). In area $\mathrm{CA} 2$, we found that plasticity is expressed prior to PNN mat- uration in CA2 (P8-11) and that degradation of PNNs enables CA2 plasticity (29), providing strong evidence that PNNs play a critical role in inhibiting a window of plasticity in CA2. Given that PNN maturation is known to be experience dependent $(29,32,33,35$, $76,77,107,108)$, we hypothesize that PNN deposition in CA2 is indicative of a developmentally regulated critical period related to hippocampal function. CA2 expresses receptors for social neuropeptides and has been implicated in social behavior $(61,65$, 109-112), and accordingly, a recent study found that PNN degradation in CA2 disrupts social memory in the adult mouse (65). We propose that this early-life window of social learning may be associated with contextual fear learning in infancy $(113,114)$, or alternatively, maternal-infant bonding $(115,116)$.

Individuals with RTT often develop seemingly normally for the first 6-18 months of age, with symptoms presenting as a regression of function after the first year of life $(68,117)$. Whether abnormalities are present prior to the onset of clinical features remains unclear. Several studies have identified differences in synaptic function in the RTT mouse model prior to the development of behavioral impairment $(19,22,54,55)$. Our data are consistent with these studies and suggest that molecular and synaptic changes are present prior to the development of behavioral deficits. Based on studies of human and rodent brain development, P7-10 in mice is approximately equivalent to $35-40$ weeks of gestation in humans, and P10-20 is similar to human infancy. Thus, our observations on CA2 plasticity and PNN maturation in the Mecp2-null mouse suggest that abnormalities in the hippocampus could occur during the window of seemingly normal development in humans. Given CA2's possible role in social behavior during development (116), abnormal PNN development in CA2 may be key in understanding the onset of social deficits that are common in RTT and are a hallmark in children with autism $(2,118,119)$.

PNN regulation by neuronal activity. PNN development is activity dependent and is modulated by normal experience in early life in several brain regions, including motor (120) and visual $(32,36,76)$ systems. In general, sensory deprivation from birth delays and attenuates PNN maturation. This raises the question: could pathological increases in hippocampal neuronal activity in RTT (78) explain the premature development of PNNs in RTT CA2? Approximately 70\% of children with RTT develop partial and generalized convulsive seizures by the age of 7 years $(1,121)$. Moreover, the Mecp2-null hippocampus is reportedly hyperexcitable and more susceptible to seizure (78). We addressed this question in our study by characterizing PNNs in an epilepsy mouse model, in which seizures develop after weaning and increase in severity with age (82). Surprisingly, we did not see changes in PNN deposition in the hippocampus until much later (>P45), after several weeks of recurrent seizure, and the direction of change was the opposite of what we expected (PNNs were reduced in CA2). We also observed a robust increase in dentate gyrus PNNs, resembling the effects of seizure activity in a mouse model of another neurodevelopmental disorder, Angelman syndrome (25), suggesting that PNN upregulation in the dentate gyrus may be a marker of frequent seizures in neurodevelopmental disorders. Consistent with our finding that seizure activity can decrease PNNs in CA2, we found that pharmacological manipulation of neuronal activity with DREADDs inversely regulated PNN deposition. Togeth- 
er, these findings indicate that aberrant neuronal activity indeed alters PNNs in the hippocampus. However, the pathological increases in activity do not appear to directly explain the persistent overexpression of PNNs in RTT CA2, and instead might suggest hypoactivity of CA2 neurons in RTT, which could lead to increases in excitability elsewhere in the hippocampus (122). Lastly, the age at which neuronal activity is altered may affect PNN regulation. We found that PNNs decreased with seizures beginning after P21, whereas another study reported that an acute seizure at P10 increased PNNs in the hippocampus (37). Further experiments will be necessary to determine the impact of aberrant neuronal activity on PNN maturation early in development versus that in late postnatal life and adulthood.

Mechanisms underlying PNN upregulation in RTT CA2. Critical-period plasticity is reportedly disrupted in several models of autism and in RTT mouse models (123). For example, Krishnan et al. discovered that onset and closure of ocular dominance plasticity were accelerated and PNNs developed precociously in area V1 of the Mecp2-null mouse (45). The accelerated time course of the critical period is attributed to the precocious maturation of $\mathrm{PV}^{+}$inhibitory interneurons $(45,47,74)$. Although we did not directly test for enhanced GABA transmission at P8-11 CA2 synapses, we did find a decrease in several genes expressed in inhibitory neurons at P10 in the Mecp2-null mouse compared with WT littermates. We speculate that the maturation of inhibitory circuitry may instead be delayed in the RTT hippocampus, based on our finding that Parv expression is lower in the Mecp2-null hippocampus at P8-11 but higher in the Mecp2-null hippocampus by P18 compared with control. Notably, a recent study found that the developmental shift in GABA is altered to a more depolarized state in $M e c p 2^{-/ Y} \mathrm{CA} 3$ pyramidal neurons at 2 postnatal weeks, providing evidence of a reduced GABAergic inhibitory tone in the hippocampus, as well as additional evidence for the presence of hippocampal impairments in early postnatal development, prior to overt signs of disease (54). Together, our findings provide evidence against a role for an accelerated maturation of inhibitory circuitry as an explanation for PNN upregulation/early closure of plasticity in RTT, and instead point to a mechanism involving CA2 pyramidal neurons.

To determine whether the increase in PNNs in RTT CA2 is dependent on a global loss of Mecp2 in the hippocampus, perhaps by way of glial cells or inhibitory network properties $(50,124)$, or is regulated in a cell-autonomous manner, we deleted Mecp2 from CA2 pyramidal neurons. Similar to our findings in the global Mecp2-null mouse, we found a greater intensity of WFA fluorescence in CA2 in comparison with 3 different controls, but no difference in intensity around $\mathrm{PNN}^{+}$neurons in CA1, CA3, or overlying somatosensory cortex. These data support the idea that Mecp2 is likely acting cell-autonomously in pyramidal neurons to upregulate PNNs in CA2. We therefore explored the expression profile of CA2-enriched genes in the Mecp2-null hippocampus. We found that the CA2-enriched gene Pcp4 was lower in the Mecp2-null hippocampus at P10. PCP4 is a known calcium modulator and therefore could be implicated in CA2's plasticity-resistant phenotype; however, its role is currently unclear $(125,126)$. The CA2-enriched gene Fgf2 was higher at both P10 and P18 in the Mecp2-null hippocampus. Interestingly, binding of the growth factor FGF-2 to its receptor requires the ECM for functional signaling $(87,127,128)$.
Whether changes in Fgf2 expression are a cause or an effect of PNN upregulation is unclear, but several studies suggest that FGF-2 may regulate ECM expression (129, 130). Moreover, FGF-2 signaling may ultimately be an important target for understanding hippocampus-dependent learning deficits exhibited in RTT $(88,89)$.

Finally, we explored one of the endogenous ECM-degrading enzymes, a matrix metalloproteinase (MMP), as a mechanism by which the loss of Mecp2 may upregulate PNNs and disrupt CA2 plasticity. MMPs function to posttranslationally modify PNNs throughout the brain and are critical for normal development and remodeling of synapses (131). Moreover, MMPs are activity-regulated in that increased neuronal activity elevates MMP expression in response to both pathological activity and learning (132-136). In particular, MMP-9 is an important regulator of structural and critical-period plasticity, and has been implicated in neurodevelopmental disorders (91). Postnatal reduction of MMP-9 levels promotes PNN formation (131), and genetic knockout of MMP-9 is sufficient to rescue behavioral deficits related to reduced PNNs in fragile X syndrome $(93,137)$. Consistent with the hypothesis that MMP-9 reduction promotes PNN formation, we found that MMP-9 protein levels were reduced in samples from Mecp2-null hippocampus, suggestive of a role of MMP regulation downstream of MeCP2.

Conclusions and implications. Taken together, these data demonstrate the existence of a window of plasticity in hippocampal area CA2 that is not observed in a mouse model of RTT. We report that PNNs appear to be abnormally high and prematurely upregulated in CA2 of an individual with RTT and in a mouse model of RTT. We identify several molecular changes related to plasticity and PNN regulation in the RTT hippocampus, such as reduced levels of the protease MMP-9. We identify PNNs as a target mechanism behind the disruption of CA2 plasticity in the RTT mouse and demonstrate that enzymatic degradation of PNNs rescues this window of CA2 plasticity in vitro. We propose that targeting aberrant PNNs in CA2 may ultimately shed light on CA2-associated behavioral function, such as social abnormalities common in Mecp2-null or Mecp2 mutant mouse models and often children with RTT $(68,138-140)$, including social recognition memory and aggression, which have been shown to be regulated by CA2 activity in mice (141). Interestingly, one study found that MeCP2 deletion from hypothalamic neurons was sufficient to produce several behavioral impairments similar to those linked to CA2 function, such as social and aggressive behaviors (142). This is particularly relevant to CA2 circuitry, as it is a major target of oxytocin- and vasopressin-expressing neurons in the paraventricular nucleus of the hypothalamus and regulates both social memory and social aggression $(109,112,143)$. Thus, future study of hippocampal area CA2 could provide further insights into to the brain circuits underlying symptoms observed with RTT, and may prove useful for development of treatment strategies.

\section{Methods}

Detailed experimental methods are outlined in Supplemental Methods.

Statistics. No specific method was used to randomize subject mice, which were chosen arbitrarily for experiments. The experimenter was blinded to Mecp2 genotype and/or treatment group in all experiments and analyses. Statistical analyses were performed using GraphPad Prism 
8 software (version 8.2.1). Comparisons between groups were analyzed using 2-tailed unpaired $t$ tests, and multigroup comparisons were analyzed using 2-way ANOVA with post hoc tests (Bonferroni's, Šidák's, or Tukey's), noted by experiment, for pairwise comparisons. Analyses were corrected for repeated measures when appropriate. $P$ value and confidence level of less than 0.05 ( $95 \%$ confidence interval) was considered significant. Unless otherwise noted, data represent mean \pm SEM.

Study approval. All procedures were approved by the National Institute of Environmental Health Sciences Animal Care and Use Committee and were in accordance with the NIH guidelines for care and use of animals. Animals in all experiments were housed under a 12-hour light/12-hour dark cycle with access to food and water ad libitum.

\section{Author contributions}

KEC, DJL, EKS, and GMA performed experiments. KEC and KEM analyzed the data. KEC, DJL, GMA, and SMD designed and coordinated the investigations. KEC wrote the manuscript with input from the other authors.

\section{Acknowledgments}

This research was supported by the Intramural Research Program of the National Institute of Environmental Health Sciences (NIE-
HS), NIH (Z01-ES100221), and an award from the International Rett Syndrome Foundation (www.rettsyndrome.org). We thank the NIEHS Molecular Genomics Core Laboratory, the Fluorescence Microscopy and Imaging Center, Heather Jensen in the Histology Core, Eli Ney at the Image Analysis Group, and the NIEHS animal care staff for their support.

Address correspondence to: Serena M. Dudek, Neurobiology Laboratory, National Institute of Environmental Health Sciences, National Institutes of Health, 111 TW Alexander Drive, MD F2-04, Research Triangle Park, North Carolina 27709, USA. Phone: 984.287.3513; Email:dudek@niehs.nih.gov.

KEC's present address is: Center for Computational Toxicology and Exposure, Environmental Protection Agency, Research Triangle Park, North Carolina, USA.

DJL and KEM's present address is: Department of Human Genetics, Emory University, Atlanta, Georgia, USA.

ES's present address is: Neuroscience Institute, Georgia State University, Atlanta, Georgia, USA.
1. Armstrong DD. Neuropathology of Rett syndrome. J Child Neurol. 2005;20(9):747-753.

2. Percy AK, et al. Rett syndrome diagnostic criteria: lessons from the Natural History Study. Ann Neurol. 2010;68(6):951-955.

3. Ebert DH, Greenberg ME. Activity-dependent neuronal signalling and autism spectrum disorder. Nature. 2013;493(7432):327-337.

4. Chahrour M, et al. MeCP2, a key contributor to neurological disease, activates and represses transcription. Science. 2008;320(5880):1224-1229.

5 . Percy AK, et al. Rett syndrome: discrimination of typical and variant forms. Brain Dev. 1987;9(5):458-461.

6. Percy AK, et al. Rett syndrome: initial experience with an emerging clinical entity. Brain Dev. 1985;7(3):300-304.

7. Schultz RJ, et al. The pattern of growth failure in Rett syndrome. Am J Dis Child. 1993;147(6):633-637.

8. Ellison KA, et al. Examination of X chromosome markers in Rett syndrome: exclusion mapping with a novel variation on multilocus linkage analysis. Am J Hum Genet. 1992;50(2):278-287.

9. Percy AK, et al. Rett syndrome: qualitative and quantitative differentiation from autism. JChild Neurol.1988;3(suppl):S65-S67.

10. Gilby KL, O'Brien TJ. Epilepsy, autism, and neurodevelopment: kindling a shared vulnerability? Epilepsy Behav. 2013;26(3):370-374.

11. Djukic A, McDermott MV. Social preferences in Rett syndrome. Pediatr Neurol. 2012;46(4):240-242.

12. Neul JL. The relationship of Rett syndrome and MECP2 disorders to autism. Dialogues Clin Neurosci. 2012;14(3):253-262.

13. Lyst MJ, Bird A. Rett syndrome: a complex disorder with simple roots. Nat Rev Genet. 2015;16(5):261-275.

14. Boggio EM, et al. Synaptic determinants of Rett syndrome. Front Synaptic Neurosci. 2010;2:28.

15. Belichenko PV, et al. Rett syndrome: 3-D confocal microscopy of cortical pyramidal dendrites and afferents. Neuroreport. 1994;5(12):1509-1513.

16. Noutel J, et al. Experience-dependent retinogeniculate synapse remodeling is abnormal in MeCP2-deficient mice. Neuron. 2011;70(1):35-42.

17. Fukuda T, et al. Delayed maturation of neuronal architecture and synaptogenesis in cerebral cortex of Mecp2-deficient mice. J Neuropathol Exp Neurol. 2005;64(6):537-544.

18. Asaka Y, et al. Hippocampal synaptic plasticity is impaired in the Mecp2-null mouse model of Rett syndrome. Neurobiol Dis. 2006;21(1):217-227.

19. Medrihan L, et al. Early defects of GABAergic synapses in the brain stem of a MeCP2 mouse model of Rett syndrome. J Neurophysiol. 2008;99(1):112-121.

20. Belichenko NP, et al. Evidence for both neuronal cell autonomous and nonautonomous effects of methyl-CpG-binding protein 2 in the cerebral cortex of female mice with Mecp2 mutation. Neurobiol Dis. 2009;34(1):71-77.

21. Tropea D, et al. Partial reversal of Rett syndrome-like symptoms in MeCP2 mutant mice. Proc Natl Acad Sci U S A. 2009;106(6):2029-2034.

22. Zhang ZW, et al. MeCP2 is required for normal development of GABAergic circuits in the thalamus. J Neurophysiol. 2010;103(5):2470-2481.

23. Gantz SC, et al. Loss of Mecp2 in substantia nigra dopamine neurons compromises the nigrostriatal pathway. J Neurosci. 2011;31(35):12629-12637.

24. Smrt RD, et al. Mecp2 deficiency leads to delayed maturation and altered gene expression in hippocampal neurons. Neurobiol Dis. 2007;27(1):77-89.

25. $\mathrm{Gu} \mathrm{B}$, et al. Ube3a reinstatement mitigates epileptogenesis in Angelman syndrome model mice. J Clin Invest. 2019;129(1):163-168.

26. Pantazopoulos H, et al. Extracellular matrix-glial abnormalities in the amygdala and entorhinal cortex of subjects diagnosed with schizophrenia. Arch Gen Psychiatry. 2010;67(2):155-166.

27. Belichenko PV, et al. Morphological study of neocortical areas in Rett syndrome. Acta Neuropathol. 1997;93(1):50-61.

28. McRae PA, Porter BE. The perineuronal net component of the extracellular matrix in plasticity and epilepsy. Neurochem Int. 2012;61(7):963-972.

29. Carstens KE, et al. Perineuronal nets suppress plasticity of excitatory synapses on CA2 pyramidal neurons. J Neurosci. 2016;36(23):6312-6320.

30. Sorg BA, et al. Casting a wide net: role of perineuronal nets in neural plasticity. J Neurosci. 2016;36(45):11459-11468.

31. Hockfield S, et al. Expression of neural proteoglycans correlates with the acquisition of mature neuronal properties in the mammalian brain. Cold Spring Harb Symp Quant Biol. 1990;55:505-514.

32. Kind PC, et al. Effects of early periods of monocular deprivation and reverse lid suture on the development of Cat-301 immunoreactivity in the dorsal lateral geniculate nucleus ( $\mathrm{dLGN})$ of the cat. J Comp Neurol. 1995;359(4):523-536.

33. Sur M, et al. Expression of a surface-associated antigen on Y-cells in the cat lateral geniculate nucleus is regulated by visual experience. J Neurosci. 1988;8(3):874-882.

34. Kind PC, et al. The development and activitydependent expression of aggrecan in the cat visual cortex. Cereb Cortex. 2013;23(2):349-360.

35. McRae PA, et al. Sensory deprivation alters aggrecan and perineuronal net expression in the mouse barrel cortex. J Neurosci. 2007;27(20):5405-5413.

36. Lander C, et al. A family of activity-dependent neuronal cell-surface chondroitin sulfate proteoglycans in cat visual cortex. J Neurosci. 1997;17(6):1928-1939.

37. McRae PA, et al. Aggrecan expression, a component of the inhibitory interneuron perineuronal 
net, is altered following an early-life seizure. Neurobiol Dis. 2010;39(3):439-448.

38. McRae PA, et al. Persistent decrease in multiple components of the perineuronal net following status epilepticus. Eur J Neurosci. 2012;36(11):3471-3482.

39. Okamoto M, et al. Kainic acid-induced convulsions cause prolonged changes in the chondroitin sulfate proteoglycans neurocan and phosphacan in the limbic structures. Exp Neurol. 2003;184(1):179-195.

40. Pizzorusso T, et al. Reactivation of ocular dominance plasticity in the adult visual cortex. Science. 2002;298(5596):1248-1251.

41. Romberg C, et al. Depletion of perineuronal nets enhances recognition memory and long-term depression in the perirhinal cortex. JNeurosci. 2013;33(16):7057-7065.

42. Lensjo KK, et al. Removal of perineuronal nets unlocks juvenile plasticity through network mechanisms of decreased inhibition and increased gamma activity. J Neurosci. 2017;37(5):1269-1283.

43. Fawcett JW, et al. The roles of perineuronal nets the perinodal extracellular matrix in neuronal function. Nat Rev Neurosci. 2019;20(8):451-465.

44. Carulli D, et al. Animals lacking link protein have attenuated perineuronal nets and persistent plasticity. Brain. 2010;133(pt 8):2331-2347.

45. Krishnan K, et al. MeCP2 regulates the timing of critical period plasticity that shapes functional connectivity in primary visual cortex. Proc Natl Acad Sci U S A. 2015;112(34):E4782-E4791.

46. Sigal YM, et al. Structural maturation of cortical perineuronal nets and their perforating synapses revealed by superresolution imaging. Proc Natl Acad Sci U S A. 2019;116(14):7071-7076.

47. Patrizi A, et al. Accelerated hyper-maturation of parvalbumin circuits in the absence of MeCP2. Cereb Cortex. 2020;30(1):256-268.

48. Guy J, et al. A mouse Mecp2-null mutation causes neurological symptoms that mimic Rett syndrome. Nat Genet. 2001;27(3):322-326.

49. Pelka GJ, et al. Mecp2 deficiency is associated with learning and cognitive deficits and altered gene activity in the hippocampal region of mice. Brain. 2006;129(pt 4):887-898.

50. Chao HT, et al. Dysfunction in GABA signalling mediates autism-like stereotypies and Rett syndrome phenotypes. Nature. 2010;468(7321):263-269.

51. Chao HT, et al. MeCP2 controls excitatory synaptic strength by regulating glutamatergic synapse number. Neuron. 2007;56(1):58-65.

52. Belichenko PV, et al. Widespread changes in dendritic and axonal morphology in Mecp2-mutant mouse models of Rett syndrome: evidence for disruption of neuronal networks. J Comp Neurol. 2009;514(3):240-258.

53. Ribeiro MC, MacDonald JL. Sex differences in Mecp2-mutant Rett syndrome model mice and the impact of cellular mosaicism in phenotype development. Brain Res. 2020;1729:146644.

54. Lozovaya N, et al. Early alterations in a mouse model of Rett syndrome: the GABA developmental shift is abolished at birth. Sci Rep. 2019;9(1):9276.

55. Cobolli Gigli C, et al. Lack of methyl-CpG bind- ing protein 2 (MeCP2) affects cell fate refinement during embryonic cortical development. Cereb Cortex. 2018;28(5):1846-1856.

56. Celio MR. Perineuronal nets of extracellular matrix around parvalbumin-containing neurons of the hippocampus. Hippocampus.

1993;3(special issue):55-60.

57. Bruckner G, et al. Region and lamina-specific distribution of extracellular matrix proteoglycans, hyaluronan and tenascin- $\mathrm{R}$ in the mouse hippocampal formation. JChem Neuroanat. 2003;26(1):37-50

58. Lendvai D, et al. Perisynaptic aggrecan-based extracellular matrix coats in the human lateral geniculate body devoid of perineuronal nets. J Neurosci Res. 2012;90(2):376-387.

59. Noguchi A, et al. Juvenile hippocampal CA2 region expresses aggrecan. Front Neuroanat. 2017;11:41.

60. Zhao M, et al. Synaptic plasticity (and the lack thereof) in hippocampal CA2 neurons. J Neurosci. 2007;27(44):12025-12032.

61. Hitti FL, Siegelbaum SA. The hippocampal CA2 region is essential for social memory. Nature 2014;508(7494):88-92.

62. Piskorowski RA, et al. Age-dependent specific changes in area CA2 of the hippocampus and social memory deficit in a mouse model of the 22q11.2 deletion syndrome. Neuron. 2016;89(1):163-176.

63. Dudek SM, et al. Rediscovering area CA2: unique properties and functions. Nat Rev Neurosci. 2016;17(2):89-102.

64. Leroy F, et al. Input-timing-dependent plasticity in the hippocampal CA2 region and its potential role in social memory. Neuron. 2017;95(5):1089-1102.

65. Dominguez S, et al. Maturation of PNN and ErbB4 signaling in area CA2 during adolescence underlies the emergence of PV interneuron plasticity and social memory. Cell Rep. 2019;29(5):1099-1112.

66. Carstens KE, Dudek SM. Regulation of synaptic plasticity in hippocampal area CA2. Curr Opin Neurobiol. 2019;54:194-199.

67. Squires KE, et al. Regulator of $\mathrm{G}$ protein signaling 14 (RGS14) is expressed pre- and postsynaptically in neurons of hippocampus, basal ganglia, and amygdala of monkey and human brain. Brain Struct Funct. 2018;223(1):233-253.

68. Chahrour M, Zoghbi HY. The story of Rett syndrome: from clinic to neurobiology. Neuron. 2007;56(3):422-437.

69. Ballas N, et al. Non-cell autonomous influence of MeCP2-deficient glia on neuronal dendritic morphology. Nat Neurosci. 2009;12(3):311-317.

70. Rakela B, et al. Astrocytic modulation of excitatory synaptic signaling in a mouse model of Rett syndrome. Elife. 2018;7:e31629.

71. Lioy DT, et al. A role for glia in the progression of Rett's syndrome. Nature. 2011;475(7357):497-500.

72. Derecki NC, et al. Wild-type microglia arrest pathology in a mouse model of Rett syndrome. Nature. 2012;484(7392):105-109.

73. Alexander G, et al. CA2 neuronal activity controls hippocampal oscillations and social behavior [preprint]. https://doi.org/10.1101/190504. Posted on bioRxiv December 28, 2017.

74. Durand S, et al. NMDA receptor regulation pre- vents regression of visual cortical function in the absence of Mecp2. Neuron. 2012;76(6):1078-1090.

75. Dityatev A, et al. Activity-dependent formation and functions of chondroitin sulfate-rich extracellular matrix of perineuronal nets. Dev Neurobiol. 2007;67(5):570-588.

76. Guimaraes A, et al. Molecular and morphological changes in the cat lateral geniculate nucleus and visual cortex induced by visual deprivation are revealed by monoclonal antibodies Cat-304 and Cat-301. J Neurosci. 1990;10(9):3014-3024.

77. Ueno $\mathrm{H}$, et al. Sensory experience-dependent formation of perineuronal nets and expression of Cat-315 immunoreactive components in the mouse somatosensory cortex. Neuroscience. 2017;355:161-174.

78. Calfa G, et al. Network hyperexcitability in hippocampal slices from Mecp2 mutant mice revealed by voltage-sensitive dye imaging. J Neurophysiol. 2011;105(4):1768-1784

79. Alexander GM, et al. CA2 neuronal activity controls hippocampal low gamma and ripple oscillations. Elife. 2018;7:e38052.

80. Rankin-Gee EK, et al. Perineuronal net degradation in epilepsy. Epilepsia. 2015;56(7):1124-1133.

81. Dityatev A. Remodeling of extracellular matrix and epileptogenesis. Epilepsia. 2010;51(suppl 3):61-65.

82. Smart SL, et al. Deletion of the K(V)1.1 potassium channel causes epilepsy in mice. Neuron. 1998;20(4):809-819.

83. Ogier M, et al. Brain-derived neurotrophic factor expression and respiratory function improve after ampakine treatment in a mouse model of Rett syndrome. J Neurosci. 2007;27(40):10912-10917.

84. Samaco RC, et al. Crh and Oprm1 mediate anxiety-related behavior and social approach in a mouse model of MECP2 duplication syndrome. Nat Genet. 2012;44(2):206-211.

85. Horvath PM, Monteggia LM. MeCP2 as an activator of gene expression. Trends Neurosci. 2018;41(2):72-74.

86. McCann KE, et al. Novel role for mineralocorticoid receptors in control of a neuronal phenotype. Mol Psychiatry. 2021;26(1):350-364.

87. Rapraeger AC, et al. Requirement of heparan sulfate for bFGF-mediated fibroblast growth and myoblast differentiation. Science. 1991;252(5013):1705-1708.

88. Stevens HE, et al. Learning and memory depend on fibroblast growth factor receptor 2 functioning in hippocampus. Biol Psychiatry. 2012;71(12):1090-1098.

89. Kang W, Hebert JM. FGF signaling is necessary for neurogenesis in young mice and sufficient to reverse its decline in old mice. JNeurosci. 2015;35(28):10217-10223.

90. Bland ST, et al. Expression of fibroblast growth factor-2 and brain-derived neurotrophic factor mRNA in the medial prefrontal cortex and hippocampus after uncontrollable or controllable stress. Neuroscience. 2007;144(4):1219-1228.

91. Reinhard SM, et al. A delicate balance: role of MMP-9 in brain development and pathophysiology of neurodevelopmental disorders. Front Cell Neurosci. 2015;9:280.

92. Wang XB, et al. Extracellular proteolysis by matrix metalloproteinase-9 drives dendritic spine enlargement and long-term potentia- 
tion coordinately. Proc Natl Acad Sci U S A. 2008;105(49):19520-19525.

93. Wen TH, et al. Genetic reduction of matrix metalloproteinase- 9 promotes formation of perineuronal nets around parvalbumin-expressing interneurons and normalizes auditory cortex responses in developing Fmr1 knock-out mice. Cereb Cortex. 2018;28(11):3951-3964.

94. Rybakowski JK, et al. Functional polymorphism of the matrix metalloproteinase- 9 (MMP-9) gene in schizophrenia. Schizophr Res. 2009;109(1-3):90-93.

95. Kim GW, et al. The role of MMP-9 in integrinmediated hippocampal cell death after pilocarpine-induced status epilepticus. Neurobiol Dis. 2009;36(1):169-180.

96. Dziembowska M, et al. High MMP-9 activity levels in fragile $\mathrm{X}$ syndrome are lowered by minocycline. Am J Med Genet A. 2013;161A(8):1897-1903.

97. Krishnan K, et al. MECP2 regulates cortical plasticity underlying a learned behaviour in adult female mice. Nat Commun. 2017;8:14077.

98. Katz DM, et al. Preclinical research in Rett syndrome: setting the foundation for translational success. Dis Model Mech. 2012;5(6):733-745.

99. Stearns NA, et al. Behavioral and anatomical abnormalities in Mecp2 mutant mice: a model for Rett syndrome. Neuroscience. 2007;146(3):907-921.

100.Shahbazian MD, et al. Balanced X chromosome inactivation patterns in the Rett syndrome brain. Am J Med Genet. 2002;111(2):164-168.

101. Kishi N, Macklis JD. MECP2 is progressively expressed in post-migratory neurons and is involved in neuronal maturation rather than cell fate decisions. Mol Cell Neurosci. 2004;27(3):306-321.

102. Rietveld L, et al. Genotype-specific effects of Mecp2 loss-of-function on morphology of layer $\mathrm{V}$ pyramidal neurons in heterozygous female Rett syndrome model mice. Front Cell Neurosci. 2015;9:145.

103. Wang IT, et al. Neuronal morphology in MeCP2 mouse models is intrinsically variable and depends on age, cell type, and Mecp2 mutation. Neurobiol Dis. 2013;58:3-12.

104.McGill BE, et al. Enhanced anxiety and stressinduced corticosterone release are associated with increased Crh expression in a mouse model of Rett syndrome. Proc Natl Acad Sci U S A. 2006;103(48):18267-18272.

105. Meng X, et al. Manipulations of MeCP2 in glutamatergic neurons highlight their contributions to Rett and other neurological disorders. Elife. 2016;5:e14199.

106.Samaco RC, et al. Female Mecp $2^{(+/)}$mice display robust behavioral deficits on two different genetic backgrounds providing a framework for pre-clinical studies. Hum Mol Genet. 2013;22(1):96-109.

107. Balmer TS, et al. Modulation of perineuronal nets and parvalbumin with developmental song learning. J Neurosci. 2009;29(41):12878-12885.

108. Ye Q, Miao QL. Experience-dependent devel- opment of perineuronal nets and chondroitin sulfate proteoglycan receptors in mouse visual cortex. Matrix Biol. 2013;32(6):352-363.

109. Smith AS, et al. Targeted activation of the hippocampal CA2 area strongly enhances social memory. Mol Psychiatry. 2016;21(8):1137-1144.

110.Stevenson EL, Caldwell HK. Lesions to the CA2 region of the hippocampus impair social memory in mice. Eur J Neurosci. 2014;40(9):3294-3301.

111. Young WS, et al. The vasopressin $1 \mathrm{~b}$ receptor is prominent in the hippocampal area CA2 where it is unaffected by restraint stress or adrenalectomy. Neuroscience. 2006;143(4):1031-1039.

112. Raam T, et al. Hippocampal oxytocin receptors are necessary for discrimination of social stimuli. Nat Commun. 2017;8(1):2001.

113. Raineki $\mathrm{C}$, et al. Functional emergence of the hippocampus in context fear learning in infant rats. Hippocampus. 2010;20(9):1037-1046.

114. Akers KG, et al. Hippocampal neurogenesis regulates forgetting during adulthood and infancy. Science. 2014;344(6184):598-602.

115. Mogi K, et al. Developmental consequences and biological significance of mother-infant bonding. Prog Neuropsychopharmacol Biol Psychiatry. 2011;35(5):1232-1241.

116. Laham BJ, et al. Newborn mice form lasting CA2-dependent memories of their mothers. Cell Rep. 2021;34(4):108668.

117. Lombardi LM, et al. MECP2 disorders: from the clinic to mice and back. J Clin Invest. 2015;125(8):2914-2923.

118. Moretti P, et al. Abnormalities of social interactions and home-cage behavior in a mouse model of Rett syndrome. Hum Mol Genet. 2005;14(2):205-220.

119. Dolce A, et al. Rett syndrome and epilepsy: an update for child neurologists. Pediatr Neurol. 2013;48(5):337-345.

120. Kalb RG, Hockfield S. Molecular evidence for early activity-dependent development of hamster motor neurons. J Neurosci. 1988;8(7):2350-2360.

121. Amir RE, et al. Rett syndrome is caused by mutations in X-linked MECP2, encoding methyl-CpG-binding protein 2. Nat Genet. 1999;23(2):185-188.

122. Boehringer R, et al. Chronic loss of CA2 transmission leads to hippocampal hyperexcitability. Neuron. 2017;94(3):642-655.

123. LeBlanc JJ, Fagiolini M. Autism: a "critical period" disorder? Neural Plast. 2011;2011:921680.

124.Alvarez-Saavedra M, et al. Cell-specific expression of wild-type MeCP2 in mouse models of Rett syndrome yields insight about pathogenesis. Hum Mol Genet. 2007;16(19):2315-2325.

125. Kleerekoper QK, Putkey JA. PEP-19, an intrinsically disordered regulator of calmodulin signaling. J Biol Chem. 2009;284(12):7455-7464.

126.Simons SB, et al. Regional differences in hippocampal calcium handling provide a cellular mechanism for limiting plasticity. Proc Natl Acad Sci U S A. 2009;106(33):14080-14084.

127. Ornitz DM. FGFs, heparan sulfate and FGFRs: complex interactions essential for development. Bioessays. 2000;22(2):108-112.

128. Duchesne L, et al. Transport of fibroblast growth factor 2 in the pericellular matrix is controlled by the spatial distribution of its binding sites in heparan sulfate. PLoS Biol. 2012;10(7):e1001361.

129. Liu JF, et al. FGF-2 and TPA induce matrix metalloproteinase-9 secretion in MCF-7 cells through PKC activation of the Ras/ERK pathway. Biochem Biophys Res Commun. 2002;293(4):1174-1182.

130. Sawaji Y, et al. Fibroblast growth factor 2 inhibits induction of aggrecanase activity in human articular cartilage. Arthritis Rheum. 2008;58(11):3498-3509.

131. Ethell IM, Ethell DW. Matrix metalloproteinases in brain development and remodeling: synaptic functions and targets. J Neurosci Res. 2007;85(13):2813-2823.

132. Szklarczyk A, et al. Matrix metalloproteinase-9 undergoes expression and activation during dendritic remodeling in adult hippocampus. JNeurosci. 2002;22(3):920-930.

133. Meighan SE, et al. Effects of extracellular matrix-degrading proteases matrix metalloproteinases 3 and 9 on spatial learning and synaptic plasticity. J Neurochem. 2006;96(5):1227-1241.

134.Wilczynski GM, et al. Important role of matrix metalloproteinase 9 in epileptogenesis. JCell Biol. 2008;180(5):1021-1035.

135. Yuan W, et al. Association between protease-specific proteolytic cleavage of brevican and synaptic loss in the dentate gyrus of kainate-treated rats. Neuroscience. 2002;114(4):1091-1101.

136. Wu YP, et al. The tissue plasminogen activator (tPA)/plasmin extracellular proteolytic system regulates seizure-induced hippocampal mossy fiber outgrowth through a proteoglycan substrate. J Cell Biol. 2000;148(6):1295-1304.

137. Sidhu H, et al. Genetic removal of matrix metalloproteinase 9 rescues the symptoms of fragile $\mathrm{X}$ syndrome in a mouse model. J Neurosci. 2014;34(30):9867-9879.

138. Moretti P, et al. Learning and memory and synaptic plasticity are impaired in a mouse model of Rett syndrome. J Neurosci. 2006;26(1):319-327.

139. Chen RZ, et al. Deficiency of methyl-CpG binding protein-2 in CNS neurons results in a Rett-like phenotype in mice. Nat Genet. 2001;27(3):327-331.

140.Gemelli T, et al. Postnatal loss of methyl-CpG binding protein 2 in the forebrain is sufficient to mediate behavioral aspects of Rett syndrome in mice. Biol Psychiatry. 2006;59(5):468-476.

141. Leroy F, et al. A circuit from hippocampal CA2 to lateral septum disinhibits social aggression. Nature. 2018;564(7735):213-218.

142. Fyffe SL, et al. Deletion of Mecp2 in Sim1expressing neurons reveals a critical role for $\mathrm{MeCP} 2$ in feeding behavior, aggression, and the response to stress. Neuron. 2008;59(6):947-958.

143. Pagani JH, et al. Role of the vasopressin $1 \mathrm{~b}$ receptor in rodent aggressive behavior and synaptic plasticity in hippocampal area CA2. Mol Psychiatry. 2015;20(4):490-499. 